\title{
Increased mRNA expression of IL-23 in the peripheral blood of patients with multiple sclerosis
}

\author{
Ali Mehdizadeh', Vahid Shaygannejad ${ }^{2 *}$, Meysam Amidfar ${ }^{3}$, Seyed Javad Hasheminia ${ }^{4}$, Mohamad Mousaei \\ Ghasroldasht ${ }^{5}$ \\ 'School of medicine, Isfahan University of medical sciences, Isfahan, Iran. \\ ${ }^{2}$ Isfahan neuroscience research center, alzahra research institute, Isfahan University of medical sciences, Isfahan, Iran. \\ ${ }^{3}$ Tehran University of medical sciences, Tehran, Iran. \\ ${ }^{4}$ Department of immunology, School of Medicine, Isfahan University of Medical Sciences, Isfahan, Iran. \\ ${ }^{5}$ Biology department, shahid ashrafi Isfahan University, clinical laboratory, alzahra hospital, Isfahan, Iran.
}

Article Info

\section{Article Notes}

Received: May 26, 2018

Accepted: July 02, 2018

\section{*Correspondence:}

Dr. Vahid Shaygannejad, Isfahan neuroscience research center, alzahra research institute, Isfahan University of medical sciences, Isfahan, Iran, Hezar-Jerib Avenue, Isfahan, Iran;

Telephone No: +98-31-36692595;

Fax No: +98-31-36688597;

Email: shaygannejad@med.mui.ac.ir

(c) 2018 Shaygannejad V. This article is distributed under the terms of the Creative Commons Attribution 4.0 International License.

\section{ABSTRACT}

Background interleukin-23 (IL-23) is a member of the IL-12 cytokine family that has shown through enhancement of T helper type 17 (Th17) cells expansion could play an important role in the inflammatory autoimmune responses in multiple sclerosis (MS)

Methods: The objective of the present study is to measure the relative expression of IL-23 mRNA in the peripheral blood of $15 \mathrm{MS}$ patients in comparison with 15 healthy control subjects.

Results: the relative gene expression level of IL-23 in the peripheral blood cells from MS Patients was significantly increased compared to healthy controls $(p<0.001)$. Conclusions Our findings revealed upregulated gene expression pattern of IL-23 in the peripheral blood of MS patients that may be a peripheral marker for diagnosis of MS and might be a novel and promising therapeutic target for MS.

\section{Introduction}

Multiple sclerosis (MS) is a chronic neuroinflammatory immunemediated disease of the central nervous system (CNS) with unknown etiology and variable clinical profile ${ }^{1}$. It has demonstrated that production of cytokines by infiltrating inflammatory cells and resident cells in the brain is involved in directing and regulating the immune response and also mediating of tissue damage ${ }^{2}$. Cytokines and their receptors are important factors of immune system and play a role in pathologic hallmarks of MS ${ }^{3,4}$. IL-23 is a heterodimeric cytokine which comprises two subunits including p19 and a common $\mathrm{p} 40$ subunit that shares with IL-125. It has demonstrated that a subset of inflammatory macrophages and dendritic cells express receptor complex of IL-236. IL-23 is constructed mainly by activation of dendritic cells (DCs) and phagocytic cells, preferentially activates memory rather than naive $T$ cells, stimulates of INF-y secretion from T cells and is involved in Th1-type immune responses ${ }^{5}$. It has found that IL-23 upon antigen-specific stimulation contribute to development and expansion of activated $\mathrm{CD}^{+}{ }^{+}$cells that produce IL-17, IL-17F, IL-6, and TNF and plays a critical role in autoimmune inflammation ${ }^{7}$. Several studies suggest that the family members of IL-17 play an important role in autoimmune and inflammatory diseases ${ }^{8}$. IL-17 is produced by Th17 cells that 
are a subset of CD4+ T-cells and IL-23 increased number of IL-17-producing CD4+ cells ${ }^{9}$. Th17 cells and IL-23 are essential for the induction of experimental models of multiple sclerosis and increased expression of IL-23 and IL-17 has been reported in patients with MS $^{7,10-14}$. Active and chronic active lesions in the multiple sclerosis have shown highly expression of proinflammatory cytokine IL23 and activated macrophages/ microglia and mature DCs have proposed as cellular sources of IL-23p19 in these lesions ${ }^{12}$. Production of $\mathrm{p} 40$ subunit of IL-23 and in vitro production of IL-23 by activated microglia support from this hypothesis that production of bioactive IL-23 plays an important role in the pathogenesis of MS ${ }^{12,15}$. IL-23 by modulation of the interaction of Microglia-T cell in multiple sclerosis may impact on the local inflammatory response in the brain ${ }^{12}$. It has suggested that correlation between expression of IL-23 and inflammatory and demyelinating lesions in MS that probably through recruitment and activation of some essential inflammatory cells that are responsible for chronic inflammation plays a crucial role in the immunopathogenesis of multiple sclerosis ${ }^{12}$. Targeting of IL-23 or IL-23- receptor has suggested as a probably promising therapeutic approach for a range of inflammatory autoimmune disease ${ }^{10}$. In the present study, we examined mRNA expression level of IL-23 using RealTime PCR technique in the peripheral blood of patients with multiple sclerosis and healthy control groups. We demonstrate herein that a high mRNA expression pattern of IL-23 in peripheral blood is a feature of MS.

\section{Material and methods}

Fifteenth patients with clinically definite MS (9 women, 6 men) from the MS Clinic of Kashani University Hospital at the Isfahan University of Medical Sciences and 15 healthy control subjects (8 females, 7 males) were included in this study. Severity of illness was evaluated using expanded disability status scale (EDSS) ${ }^{16}$ and disease type was defined as relapsing-remitting (RR), primary progressive (PP) or secondary progressive (SP). All of patients were diagnosed according to the revised McDonald criteria ${ }^{17}$, and MS clinical forms were determined according to the classification of Lublin \& Reingold ${ }^{18}$.Ten patients $(66.7 \%)$ suffered from relapsing-remitting MS; three patients (20\%) were in the primary progressive phase of the disease and two patients $(13.3 \%)$ were in the secondary progressive phase of the disease. None of the Patients was received any immunomodulatory drugs such as methylprednisolone or
interferon-(IFN- $\beta$ ) for at least 4 weeks prior to inclusion, and had not had an acute exacerbation prior to inclusion. The Ethical Committee on Human Research, Isfahan University of Medical Sciences approved protocol of study and written informed consent was obtained from all participating prior to entering the study. Patients with infection diseases or other autoimmune diseases, cardiovascular or renal disease, cancer and blood systemic disorders were excluded from study. Clinical and demographic features of participants have been summarized in Table 1.

Venous blood samples ( $5 \mathrm{ml}$ ) were collected from both control and patient groups and Total RNA was extracted from whole blood using using High pure RNA isolation kit according to the manufacturer's instructions (Qiagen, USA). Spectrophotometry showed optical density ratio of $260 \mathrm{~nm}$ to $280 \mathrm{~nm}$ (1.8-2.1) for extracted RNA confirming purity and quantity of extracted RNA in optimal range. Complementary DNA (cDNA) was synthesized with oligo (dT) primer using a reverse transcription ki (Fermentaz, Germany). IL-23 mRNA expression was quantified by realtime PCR using Prime Q-Mastermix reagent kit (2X, Realtime PCR with SYBR Green I) (GenetBio, republic of Korea). All reactions were performed at triplicate. The relative gene expression of the IL-23 was normalized to GAPDH as the reference gene.

\section{Statistical analysis}

The normal distribution of clinical variables and gene expression data were checked using Kolmogorov-Smirnov test. Two-tailed t-test, chi-square test, Kruskal-Wallis test and Mann-Whitney test were used for comparison of study groups by SPSS20. The relationships between IL-23 mRNA and clinical characteristics were examined with Pearson's correlation coefficient. $\mathrm{p}<0.05$ was considered as statistical significant.

\section{Results}

Demographic characteristics didn't show significant difference between two groups. Two-tailed t-test showed significantly higher relative levels of IL-23 mRNA (7.98 $\pm 1.61, t=-16.693, p<0.001)$ in peripheral blood of patients with multiple sclerosis in compared to healthy controls (Figure 1). We didn't find significant correlation between mRNA expressions of IL-23 and severity of illness in MS patients ( $\mathrm{rs}=-0.065, \mathrm{p}=0.817$ ). A significant negative correlation was found between severity of illness and age in patients with MS ( $r s=-0.731, p=0.002)$. Multiple sclerosis

Table 1: Demographic characteristic of participants

\begin{tabular}{|l|c|c|c|c|}
\hline Characteristics & $\begin{array}{c}\text { Controls } \\
\mathrm{N}=15\end{array}$ & $\begin{array}{c}\text { MS } \\
\text { group, } \mathrm{n}=15\end{array}$ & $t$-test/X2 & Significance, $p$ \\
\hline Age (SD), $\mathrm{y}$ & $46.40(18.34)$ & $36.60(6.33)$ & $t(d f=28)=1.95$ & 0.061 \\
\hline Gender, M/F & $7 / 8$ & $6 / 9$ & $\mathrm{X}^{2}=0.136$ (Fisher exact) & 0.713 \\
\hline EDSS score & - & $6.66(1.23)$ & - & - \\
\hline
\end{tabular}




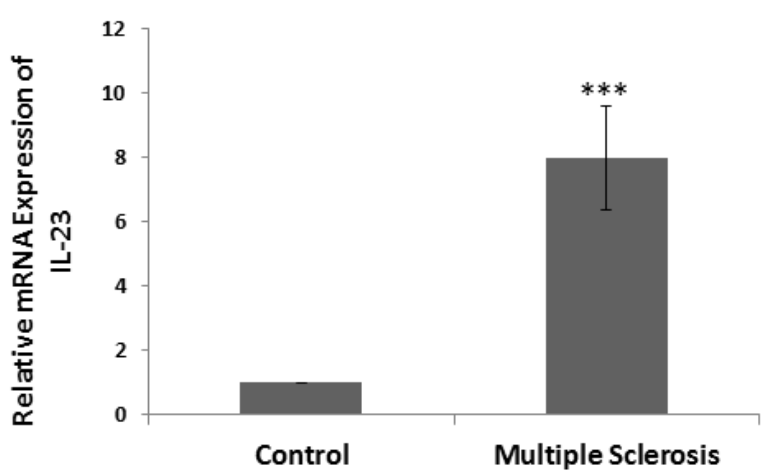

Figure 1: IL-23 mRNA expression in peripheral blood of healthy controls and patients with multiple sclerosis. The relative gene expression of IL-23 was $7.98 \pm 1.61$ in MS patients. The relative levels of IL-23 mRNA expression was significantly increased in MS patients when compared to healthy controls $(p=0.000)$. *** The mean difference is significant $(p<0.001)$.

patients showed no significant correlation between age and IL-23 mRNA level ( $r s=0.148, p=0.599)$. Two-tailed t-test revealed no significant difference in the mRNA expression level of IL-23 between male and female ( $p=0.493$ ).

A Kruskal-Wallis test showed that there was a statistically significant difference in the relative levels of IL23 mRNA between three groups $\left(\chi^{2}=7.33, \mathrm{df}=2, \mathrm{p}=0.026\right)$.

Mann-Whitney test showed significantly higher relative levels of IL-23 mRNA in peripheral blood of RRMS patients in compared to PPMS patients $(\mathrm{Z}=-2.20, \mathrm{p}=0.028)$. Relative levels of IL-23 mRNA in peripheral blood didn't show a significant difference between RRMS patients and SPMS patients $(\mathrm{Z}=-1.937, \mathrm{p}=0.053)$ as well as between PPMS patients and SPMS patients $(Z=-0.577, p=0.564)$.

\section{Discussion}

In the present study we found higher mRNA expression of IL-23 gene in peripheral blood of MS patients in compared to control subjects. Our results are in agreement with findings reported by several studies indicating increased plasma and mRNA expression levels of IL-23 in MS patients ${ }^{12,14,19,20}$. It has found that MS patients show increased secreted levels of IL-23 from monocyte-derived Dendritic Cells (DCs) and also mRNA overexpression of both IL-23p19 and IL-12/23p40 ${ }^{14}$. It has postulated that IL-23 by affecting of immune system through polarization of the innate immune response toward a Th1 bias may plays an important role in the pathogenesis of Multiple sclerosis as a Th1 type $\mathrm{T}$ cell mediated inflammatory autoimmune and demyelinating disease of the $\mathrm{CNS}^{14,21-}$ 23. Proinflammatory cytokine IL-23 plays a key role in chronic inflammatory autoimmune responses and IL-23deficient mice have shown a resistance to induction of experimental autoimmune encephalomyelitis (EAE) ${ }^{10}$. In addition, it has found that MS patients have significant higher plasma level of IL-23 when compared to healthy subjects $^{20}$. A significantly higher serum and CSF levels of IL-23 and positive correlation of IL-23 with IL-17 and with the poor outcome of MS have been reported in MS patients, which suggest IL-23 as a probably sensitive and stable marker for development of MS and therapeutic targets for $\mathrm{MS}^{24}$.The ability of IL-23 in expansion of T helper type 17 (Th17) cells plays a crucial role in the development and the maintenance of autoimmune inflammation ${ }^{25}$. Stopping of organ-specific autoimmune inflammation by specific inhibition of the IL-23/IL-17 immune axis in the mouse suggest that specific blockade of the IL23 immune pathway in humans may be an efficient and undamaged treatment for inflammatory diseases mediated by immune system dysfunction ${ }^{26}$. Moreover, MS patients have demonstrated increased amounts of IL-12p40 in the nervous system and increased production of IL-12 by PBMC $^{5,27,28}$. Analysis of mRNA expression levels of IL-23 in PBMCs of untreated MS patients showed increased mRNA expression of IL-23 that treatment with IFN- $\beta$ induced suppression of IL-23 mRNA levels ${ }^{19}$. Investigation on the expression of IL-23 in brain tissues of MS patients using in situ hybridization (ISH) and immunohistochemistry showed highly expression of IL-23p19 in active and chronic active lesions ${ }^{12}$. It has demonstrated that activated macrophage/microglia produce p19 subunit of IL-23 in the lesions in multiple sclerosis ${ }^{12}$. In addition, reported findings related to production of p40 subunit in these lesions as well as in vitro evidence about production of IL-23 in activated microglia, powerfully verifies this hypothesis that production of bioactive IL-23 by microglia plays a critical role in the pathogenesis of $\mathrm{MS}^{12}$, 15. Moreover, active and chronic active lesions have clearly demonstrated highly mRNA and protein expression of p19 subunit of IL-23 in macrophages/microglia in, but silent lesions and normal brain sections showed low or even undetectable expression ${ }^{12}$. Association between IL-23 expression and inflammatory and demyelinating lesions suggesting IL-23 as a probably crucial factor that plays an important role in the immunopathogenesis of multiple sclerosis through recruitment and activation of variety of inflammatory cells involved in the induction of chronic inflammation ${ }^{12}$. Collected data in mice confirm that in the development of EAE the IL-23 plays an important role rather than $\mathrm{IL}-12^{10,29}$.It has found that therapeutic effect of IL-23 induced by anti-IL-23p19 inhibited relapse of disease during active $\mathrm{EAE}^{26}$. Some mechanisms of action have suggested for anti-IL-23p19 therapeutic inhibition of EAE relapse including inhibition of epitope spreading to additional encephalitogenic epitopes such as proteolipid protein178-191 (PLP178-191) and recruitment of newly activated pathogenic $\mathrm{T}$ cells and reduction of the number of inflammatory myeloid cells invading the CNS and diminishing the levels of monokines secreted by 
macrophage and dendritic cells ${ }^{26}$.It has suggested that reduced number of inflammatory myeloid cells that invade the CNS and diminish the concentration of monokines produced by dendritic cells and macrophage such as IL-1, IL-6, and TNF implicate in the mechanism of action of antiIL-23p19 therapy ${ }^{26}$. The pathological hallmarks of EAE and MS have attributed to this myeloid cell invasion and production of proinflammatory factors ${ }^{26}$. It has proposed that the inhibition of IL-23 using designing drugs that target the IL-23p19 or IL-23 receptor might be a novel and promising therapeutic strategy in treatment of autoimmune inflammatory diseases such as multiple sclerosis ${ }^{25,26}$. The main finding of our study is that untreated MS patients have increased expression of IL-23 mRNA in the peripheral blood that is agreement with findings reported by previous

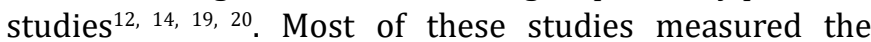
common IL-12p40 subunit shared by IL-12 and IL-23. It has demonstrated that patients with both RR and SP MS show overexpression of IL-23p19 and p40, but not IL-12p35 mRNA and also increased IL-23 production compared to healthy subjects ${ }^{14}$. Accordingly, increased mRNA levels of IL-23 have been found in MS ${ }^{12,13}$. It has demonstrated that IL-23 deficient mice are resistant to EAE animal models and untreated MS patients have shown increased levels of IL-23 mRNA that treatment with IFN- $\beta$ result in suppression of IL-23 mRNA $^{10,19}$. In conclusion, we have presented findings that peripheral blood from patients with multiple sclerosis exhibit higher mRNA expression of proinflammatory cytokine IL-23 when compared to healthy controls. These findings support the role of IL-23 in the pathogenesis of multiple sclerosis as an inflammatory autoimmune disease and may have implications as a specific peripheral marker for MS.

\section{References}

1. Compston A, Lassmann H, McDonald I. The story of multiple sclerosis. McAlpine's Multiple Sclerosis London: Churchill Livingstone. 2006; 3-68.

2. Brosnan CF, Raine CS. Mechanisms of immune injury in multiple sclerosis. Brain Pathology. 1996; 6: 243-257.

3. Bjartmar C, Wujek J, Trapp B. Axonal loss in the pathology of MS: consequences for understanding the progressive phase of the disease. Journal of the neurological sciences. 2003; 206: 165-171.

4. Lucchinetti C, Bruck W, Parisi J, et al. Heterogeneity of multiple sclerosis lesions: implications for the pathogenesis of demyelination. Annals of neurology. 2000; 47: 707-717.

5. Oppmann B, Lesley R, Blom B, et al. Novel p19 protein engages IL12 p40 to form a cytokine, IL-23, with biological activities similar as well as distinct from IL-12. Immunity. 2000; 13: 715-725.

6. Parham $\mathrm{C}$, Chirica $\mathrm{M}$, Timans J, et al. A receptor for the heterodimeric cytokine IL-23 is composed of IL-12R $\beta 1$ and a novel cytokine receptor subunit, IL-23R. The Journal of Immunology. 2002; 168: 5699-5708.

7. Langrish CL, Chen Y, Blumenschein WM, et al. IL-23 drives a pathogenic $\mathrm{T}$ cell population that induces autoimmune inflammation. Journal of Experimental Medicine. 2005; 201: 233-240.

8. Kolls JK, Lindén A. Interleukin-17 family members and inflammation. Immunity. 2004; 21: 467-476.

9. Hoeve MA, Savage ND, de Boer T, et al. Divergent effects of IL-12 and
IL-23 on the production of IL-17 by human T cells. European journal of immunology. 2006; 36: 661-670.

10. Cua DJ, Sherlock J, Chen Y, et al. Interleukin-23 rather than interleukin-12 is the critical cytokine for autoimmune inflammation of the brain. Nature. 2003; 421: 744.

11. Gran B, Zhang GX, Yu S, et al. IL-12p35-deficient mice are susceptible to experimental autoimmune encephalomyelitis: evidence for redundancy in the IL-12 system in the induction of central nervous system autoimmune demyelination. The Journal of Immunology 2002; 169: 7104-7110.

12. Li Y, Chu N, Hu A, et al. Increased IL-23p19 expression in multiple sclerosis lesions and its induction in microglia. Brain. 2006; 130: 490501.

13. Matusevicius D, Kivisäkk P, He B, et al. Interleukin-17 mRNA expression in blood and CSF mononuclear cells is augmented in multiple sclerosis. Multiple Sclerosis Journal. 1999; 5: 101-104.

14. Vaknin-Dembinsky A, Balashov K, Weiner HL. IL-23 is increased in dendritic cells in multiple sclerosis and down-regulation of IL-23 by antisense oligos increases dendritic cell IL-10 production. The Journal of Immunology. 2006; 176: 7768-7774.

15. Windhagen A, Newcombe J, Dangond F, etal. Expression of costimulatory molecules B7-1 (CD80), B7-2 (CD86), and interleukin 12 cytokine in multiple sclerosis lesions. Journal of Experimental Medicine. 1995; 182: 1985-1996.

16. Kurtzke JF. Rating neurologic impairment in multiple sclerosis: an expanded disability status scale (EDSS). Neurology. 1983; 33: 14441444.

17. Polman $\mathrm{CH}$, Reingold SC, Banwell B, et al. Diagnostic criteria for multiple sclerosis: 2010 revisions to the McDonald criteria. Annals of neurology. 2011; 69: 292-302.

18. Lublin FD, Reingold SC. Defining the clinical course of multiple sclerosis results of an international survey. Neurology. 1996; 46: 907-911.

19. Krakauer M, Sorensen P, Khademi M, et al. Increased IL-10 mRNA and IL-23 mRNA expression in multiple sclerosis: interferon- $\beta$ treatment increases IL-10 mRNA expression while reducing IL-23 mRNA expression. Multiple Sclerosis Journal. 2008; 14: 622-630.

20. Shajarian M, Alsahebfosoul F, Etemadifar M, et al. IL-23 plasma level measurement in relapsing remitting multiple sclerosis (RRMS) patients compared to healthy subjects. Immunological investigations. 2015; 44: 36-44.

21. Martin R, McFarland HF, McFarlin DE. Immunological aspects of demyelinating diseases. Annual review of immunology. 1992; 10: 153-187.

22. Sospedra M, Martin R. Immunology of multiple sclerosis. Annu Rev Immunol. 2005; 23: 683-747.

23. Weiner HL. Multiple sclerosis is an inflammatory T-cell-mediated autoimmune disease. Archives of Neurology. 2004; 61: 1613-1615.

24. Wen SR, Liu GJ, Feng RN, et al. Increased levels of IL-23 and osteopontin in serum and cerebrospinal fluid of multiple sclerosis patients. Journal of neuroimmunology. 2012; 244: 94-96.

25. Tang $\mathrm{C}$, Chen $\mathrm{S}$, Qian $\mathrm{H}$, et al. Interleukin-23: as a drug target for autoimmune inflammatory diseases. Immunology. 2012; 135: 112-124.

26. Chen Y, Langrish CL, Mckenzie B, et al. Anti-IL-23 therapy inhibits multiple inflammatory pathways and ameliorates autoimmune encephalomyelitis. The Journal of clinical investigation. 2006; 116: 1317-1326.

27. Kopp T, Lenz P, Bello-Fernandez C, et al. IL-23 production by cosecretion of endogenous p19 and transgenic p40 in keratin 14/p40 transgenic mice: evidence for enhanced cutaneous immunity. The Journal of Immunology. 2003; 170: 5438-5444.

28. Lankford CS, Frucht DM. A unique role for IL-23 in promoting cellular immunity. Journal of leukocyte biology. 2003; 73: 49-56.

29. Becher B, Durell BG, Noelle RJ. IL-23 produced by CNS-resident cells controls $\mathrm{T}$ cell encephalitogenicity during the effector phase of experimental autoimmune encephalomyelitis. The Journal of clinical investigation. 2003; 112: 1186-1191. 